Available online at www.sciencedirect.com

www.cya.unam.mx/index.php/cya

Contaduría y Administración 60 (S1) 82-106

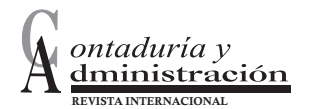

www.contaduriayadministracionunam.mx/

\title{
Factores críticos de éxito para el despliegue del mantenimiento productivo total en plantas de la industria maquiladora para la exportación en Ciudad Juárez: una solución factorial
}

\author{
Critical success factors for the strategic deployment of total \\ productive maintenance in the exporting maquiladora industry \\ in Ciudad Juárez: a factorial solution
}

\author{
Andrés Hernández Gómez ${ }^{\mathrm{a}}$, Carlos Escobar Toledo ${ }^{\mathrm{b}}$, \\ Juan M. Larios Prado b,*, Salvador Noriega Morales ${ }^{\mathrm{a}}$
}

${ }^{a}$ Universidad Autónoma de Ciudad Juárez, Departamento de Ingeniería Industrial, México

${ }^{b}$ Universidad Nacional Autónoma de México

Recibido el 23 de mayo de 2014; aceptado el 5 de septiembre de 2014

Disponible en internet el 14 de Septiembre de 2015

\section{Resumen}

En este artículo se presentan los resultados de un estudio efectuado a personal relacionado con el proceso de implantación del Mantenimiento Productivo Total (MPT) en empresas de la industria maquiladora de Ciudad Juárez, Chihuahua, México. Se aplicó un Análisis Factorial para identificar los Factores Críticos de Éxito (FCE) vinculados con la

\footnotetext{
"Autor para correspondencia

Correo electrónico: jlariosp@ hotmail.com, juan_larios2001@yahoo.com.mx (J. M. Larios)

La revisión por pares es responsabilidad de la Universidad Nacional Autónoma de México.

http://dx.doi.org/10.1016/j.cya.2015.08.005

0186-1042/Derechos Reservados (C) 2015 Universidad Nacional Autónoma de México, Facultad de Contaduría y Administración. Este es un artículo de acceso abierto distribuido bajo los términos de la Licencia Creative Commons CC BY-NC-ND 4.0.
} 
implantación del MPT. El artículo inicia con la revisión del estado del arte que se llevó a cabo para la identificación de los FCE, los cuales están organizados en aspectos estratégicos y administrativos, del desarrollo del recurso humano y técnicos propios del MPT. Con esta información se construyó un instrumento para determinar los FCE en las plantas. La tercera sección describe la metodología, se valida el cuestionario y se justifica la aplicación de las pruebas estadísticas y el Análisis Factorial. La cuarta sección presenta los resultados, iniciando con la descripción del muestreo, conformado por 306 sujetos vinculados con la implantación del MPT. Se identificaron nueve factores críticos de éxito relacionados con la implantación efectiva del MPT, y se discuten sus efectos.

Derechos Reservados@2015 Universidad Nacional Autónoma de México, Facultad de Contaduría y Administración.

Este es un artículo de acceso abierto distribuido bajo los términos de la Licencia Creative Commons CC BY-NC-ND 4.0.

Palabras clave: Factores críticos de éxito; Mantenimiento productivo total; Análisis factorial

\section{Abstract}

This paper presents the findings of a research project applied to personnel involved in the implementation process of the Total Productive Maintenance (TPM) in manufacturing companies of the twin plant industry in Ciudad Juárez, Chihuahua, México. Its purpose is the application of Factorial Analysis to identify the Critical Success Factors (CSF) for the effective deployment of the TPM. The first section summarizes the review of the state of the art done for the identification of CSF, they are organized by their affinity to strategic and management issues, human resources and technical-operational topics of TPM; with this information a questionnaire was developed for the determination of the CSF in the plants. The third section is about the methodology, explaining the questionnaire test, its validation and application, also statistical tests are discussed. The fourth section is about the findings, the first part describes the sample and the sampling process, it also explains the application to a sample of 306 knowledgeable persons working with TPM. Nine critical success factors related to the effective implementation of the TPM were identified.

All Rights Reserved (C) 2015 Universidad Nacional Autónoma de México, Facultad de Contaduría y Administración.

This is an open access item distributed under the Creative Commons CC License BY-NCND 4.0.

Keywords: Critical success factors; Total productive maintenance; Factorial analysis

\section{Introducción}

El mantenimiento fue considerado durante mucho tiempo como una actividad de índole operativa, escasamente vinculada con el sistema administrativo de las organizaciones, sin embargo esta concepción ha cambiado. En el ambiente de una 
economía global, la administración del mantenimiento es una función clave que soporta las operaciones de producción de empresas manufactureras. En particular, para Muchiri (2011) el mantenimiento es la combinación de actividades de índole técnica y administrativa requeridas para mantener el equipo, las instalaciones y otros activos fijos en condiciones deseables de operación, o para restaurarlos hacia esta condición, lo cual se despliega como estrategia a través del MPT y así constituye una fuente de competitividad. Ahuja y Kamba (2008a) coinciden en su carácter estratégico, puesto que se trata de una función de vital importancia para el negocio porque incrementa la competitividad de la organización al reducir las quejas de los clientes y los niveles de inventario, aumenta la tasa de calidad de la producción, mejora el tiempo de entrega e incrementa la moral y la seguridad del empleado, lo que finalmente impacta en las ventas y la rentabilidad (Park y Han, 2001; Ahmed et al., 2005; Ahuja y Khamba, 2008b; Thomas et al., 2010). A pesar de que estas razones explican la importancia del MPT, es necesario investigar los factores que inciden en su efectividad, dado que existe evidencia empírica de que en plantas de la industria maquiladora no ha sido efectiva su implantación, y porque en la literatura no se observa una teoría con suficiente poder explicativo. Este artículo presenta la teoría de los Factores Críticos de Éxito para la implantación estratégica del MPT, continúa con la descripción de la metodología, describe los resultados y finaliza con su discusión.

\section{Revisión de literatura}

Esta sección es una breve discusión de la teoría y se comentan varios tópicos del MPT para explicarlo y caracterizarlo: inicia con los conceptos básicos del MPT y su relación con otras prácticas de manufactura, continúa con la inclusión del MPT en la teoría de la Estrategia de la Manufactura (EM), sus relaciones con las Estrategias de Innovación y Desarrollo Tecnológico (EIDT) y la Manufactura Esbelta (ME), posteriormente se discute el efecto del MPT en la competitividad como una forma de medir el éxito de su implantación, y finalmente se enlistan los FCE obtenidos en la revisión de la literatura.

\section{Mantenimiento Productivo Total}

Teeravaraprug et al. (2011) afirman que el MPT es una estrategia para responder a la demanda de un mercado competitivo en costo y calidad, estrategia que para Nakajima (1988) se despliega a través del mejoramiento de la efectividad del equipo, el establecimiento de un sistema de mantenimiento productivo, la implantación por varios departamentos, el involucramiento de los empleados y la promoción a 
través de actividades autónomas realizadas por grupos pequeños; debido a estas características, para Thomas et al. (2010) el MPT es una función de manufactura diseñada para maximizar la efectividad del equipo.

\section{Relación del MPT con otras iniciativas de mejora}

En la literatura existen diversos estudios sobre la implantación y efectividad del MPT relacionados con su aplicación junto a otras iniciativas, como la Administración Total de la Calidad (ATC), el Justo a Tiempo (JAT) y el Seis Sigma (SS). Por ejemplo, Al-Hassan et al. (2000) afirman que cuando se establece primero la ATC es mas rápida la implantación efectiva del MPT, si bien Konecny y Thun (2011) reportan que cuando se implanta primero el MPT, el desempeño operacional es mejor que cuando se implantan concurrentemente la ATC y el MPT, lo cual se puede explicar debido a que el MPT es un subconjunto de la ATC, según Park y Han (2001). Respecto al JAT, Thomas et al. (2010) afirman que la efectividad es mayor cuando el MPT se implanta previamente, lo que contrasta con el reporte de Cua et al. (2001) de que las empresas que han implantado conjuntamente la ATC, el JAT y el MPT tienen mejores indicadores de desempeño operacional, aunque no explican la forma para administrarlos con efectividad y, por otra parte, con el despliegue de Seis Sigma, Thomas y Gareth (2007) contribuyen a la controversia, ya que recomiendan la combinación del MPT con Seis Sigma porque también impacta positivamente en dichos indicadores. En lo que se refiere a la Manufactura Esbelta (ME), según Shah y Ward (2003) integra diversas técnicas, entre ellas el Justo a Tiempo, los Sistemas de Calidad, los Equipos de Trabajo, la Manufactura Celular y el Mantenimiento Productivo Total bajo una implantación concurrente. Finalmente, se rescata que estas técnicas han sido aplicadas ampliamente con resultados cuestionables (Bruun y Mefford, 2004), y constituyen una teoría razonable pero controversial.

\section{Relación entre la EM, la EIDT y el MPT}

Hay dos niveles de integración del MPT con las Estrategias de Manufactura (EM) y la de Innovación y Desarrollo Tecnológico (EIDT): el nivel estratégico y el nivel táctico. Bajo el primero, la Estrategia Competitiva se despliega a través de la EM y la EIDT, que convergen en el desarrollo de tecnologías propias para el diseño de equipo, con el propósito de que sus características técnicas no sean imitables por los competidores (Martínez y Escobar, 2011), incluyendo tecnologías blandas de desarrollo interno como el MPT; en el mismo sentido, Avella et al. (2001), Dangayach y Deshmukh (2006) y Gyampah y Acquaah (2008) afirman que la innova- 
ción es una prioridad competitiva porque los proyectos de innovación y desarrollo tecnológico impulsan el crecimiento de técnicas para la producción, como el MPT. En este esquema, el MPT es una tecnología blanda y elemental de la estrategia, mientras que a nivel táctico y operacional, el MPT se aplica para la adaptación o el mejoramiento de equipos genéricos y el desarrollo de competencias de manufactura (Avella et al., 2001) y, de acuerdo con Rho et al. (2001), para mejorar el desempeño organizacional; en lo táctico se puede considerar que el MPT es un medio para desplegar las EM y la de EIDT y, para que sea efectivo, es importante que exista consistencia entre la EM adoptada y las prácticas de manufactura.

De esta discusión se puede concluir que existe cierta ambigüedad en la relación de las estrategias, lo táctico y operacional con el MPT, según Houshmand y Jamshidnezhad (2006), una posible explicación es que la teoría de los sistemas de producción carece de fundamentos científicos, ya que está constituida por conocimientos empíricos, del tipo de tecnologías operativas (Bunge, 2004); en el mismo sentido Suh (1990) afirma que la aplicación de estos sistemas de manufactura responde más al desarrollo tecnológico como fuente de competitividad que a teorías derivadas del avance científico, por lo que la ciencia de los sistemas de manufactura ha quedado rezagada respecto al desarrollo de estos mismos sistemas. Estas razones justifican la necesidad de estudiar el proceso de implantación del sistema del MPT desde una perspectiva científica, con objeto de aumentar el poder explicativo de la teoría relacionada con los factores críticos involucrados en su despliegue exitoso, y para ese propósito se discute la teoría del campo en las siguientes secciones.

\section{Relación del MPT con la competitividad}

La intensificación de la rivalidad en la economía globalizada, la amenaza de la competencia global, la búsqueda de rentabilidad (Pintelon et al., 2006), además de la demanda cambiante de los mercados y los rápidos desarrollos tecnológicos (Ahuja y Khamba, 2007), ejercen fuertes presiones en las organizaciones y las impulsan a la implantación de iniciativas para incrementar la productividad, como son la Manufatura Esbelta o Seis Sigma. Aunque también hay que considerar otros factores de cambio, como el incremento en la eficiencia y la especialización interna, para satisfacer los requisitos de flexibilidad y entrega de productos al mercado (Yamashina, 1995). En virtud de estas presiones y en la búsqueda de competitividad, las empresas se ven obligadas a mejorar su desempeño organizacional (Ahuja y Khamba, 2008b) desarrollando sus competencias de manufactura (Hayes y Wheelwright, 1984; Avella et al., 2001; Dangayach y Deshmukh, 2006; Theodorou y Florou, 2008), y son estas condiciones las que impulsan la implantación del MPT porque es una fuente de ahorro y de ventaja competitiva (Chen y Meng, 2011), 
su efectiva administración contribuye al desempeño superior y es una importante fuente de competitividad (Garg y Deshmukh, 2006; Muchiri, 2011).

De manera estructural esto implica que el MPT es un predictor del desempeño organizacional con diversos efectos, entre ellos los financieros (Brah y Chong, 2004), el desarrollo de competencias de manufactura (Ahuja y Kamba, 2008a) y según Swanson (2001) sus efectos operacionales se observan en la mejora de cuatro prioridades competitivas — costo, calidad, tiempo de entrega y flexibilidad de la mezcla del producto- - y con estos mismos efectos se miden los resultados de su implantación. Esta relación, de tipo predictor, es la que permite vincular al MPT con la efectividad, como lo reportan varios autores (McKone et al., 2001; Cua et al., 2001; Konecny y Thun, 2011). Por lo anterior se deduce que el MPT incide en la efectividad del sistema de producción, lo cual constituye la oportunidad para investigar los factores que impactan al desempeño operacional bajo el paradigma de los FCE. En la siguiente sección se exponen los FCE identificados en la revisión de la literatura.

\section{Factores Críticos del Éxito del MPT}

En general, los factores críticos del éxito son áreas que aseguran un desempeño competitivo (Leidecker y Bruno, 1984), esenciales para el logro de la misión (Caralli, 2004). Rockart y Bullen (1981) afirman que su propósito es centrar la atención en lo prioritario, como son las estrategias organizacionales (Caralli, 2004; Plant y Willcocks, 2007). En la revisión de la literatura referente a los factores relacionados con el éxito en la implantación del MPT, algunos autores los consideran FCE, mientras que otros no hacen tal distinción, solo son mencionados como actividades observadas durante el proceso de implantación, por ejemplo García et al. (2012) estudian las actividades administrativas que pueden influir en el éxito del MPT. De la revisión de la literatura se determinaron los factores, clasificados por su afinidad en tres categorías que se resumen en las Tablas 1, 2 y 3 . En la Tabla 1 se encuentran los factores identificados con aspectos relacionados al proceso de planeación estratégica; en la Tabla 2 se muestran los FCE vinculados con los aspectos técnicos del MPT y, por último, en la Tabla 3 se enlistan los relacionados con el desarrollo del recurso humano.

En la Tabla 1 se agruparon 11 factores en los que se incluyen actividades relacionadas con la alineación estratégica del MPT en la estrategia corporativa, las contribuciones gerenciales requeridas para el despliegue del MPT, la asignación y despliegue de recursos y la comunicación previa y durante el proceso de implantación; se sigue la organización presentada por García et al. (2012). 
Tabla 1.

Factores relacionados con la planeación estratégica.

\begin{tabular}{|c|c|}
\hline Factores identificados & Autores \\
\hline Asesoría de Staff especializado & Swanson (1997). \\
\hline $\begin{array}{l}\text { Compromiso / contribución de la alta } \\
\text { administración }\end{array}$ & $\begin{array}{l}\text { Ahuja y Kamba (2008c), Ahmed et al. (2005), Chan } \\
\text { et al. (2005), Cooke (2000), Bamber et al. (1999), } \\
\text { Hansson y Backlund (2003), Kumar et al. (2006), } \\
\text { Lycke (2003), Lazim y Ramayah (2010), McAdam } \\
\text { y McGeough (2000), Park y Han (2001). }\end{array}$ \\
\hline Transformaciones culturales & $\begin{array}{l}\text { Ahuja y Kamba (2008c), Carannante et al. (1996), } \\
\text { Cooke (2000), Eti et al. (2004), Park y Han (2001). }\end{array}$ \\
\hline $\begin{array}{l}\text { Despliegue de políticas proactivas de } \\
\text { mantenimiento }\end{array}$ & Ahuja y Kamba (2008c), Carannante et al. (1996). \\
\hline $\begin{array}{l}\text { Planeación Corporativa / plan de } \\
\text { implementación }\end{array}$ & $\begin{array}{l}\text { Brah y Chong (2004), Alsyouf (2006), Chand y } \\
\text { Shirvani (2000), Bamber et al. (1999), Eti et al. } \\
\text { (2004), Davis (1997), Graisa y Al-Habaibeh (2011), } \\
\text { Jonsson (1997), Lazim y Ramayah (2010), Park y } \\
\text { Han (2001). }\end{array}$ \\
\hline Liderazgo de la alta administración & $\begin{array}{l}\text { Brah y Chong (2004), Fredendall et al. (1997), Lycke } \\
\text { (2003), Lazim y Ramayah (2010). }\end{array}$ \\
\hline Proceso de sensibilización / comunicación & $\begin{array}{l}\text { Chan et al. (2005), Bamber et al. (1999), Eti et al. } \\
\text { (2004), Lycke (2003), Park y Han (2001). }\end{array}$ \\
\hline $\begin{array}{l}\text { Selección efectiva del equipo de } \\
\text { implementación }\end{array}$ & Chan et al. (2005), Lycke (2003). \\
\hline Introducción gradual del MPT & Chan et al. (2005), Fredendall et al. (1997). \\
\hline $\begin{array}{l}\text { Alineación de objetivos organizacionales con } \\
\text { el MPT }\end{array}$ & Cooke (2000), Bamber et al. (1999), Jonsson (1997). \\
\hline $\begin{array}{l}\text { Asignación de recursos (financieros, humanos, } \\
\text { tiempo, sistema de recompensas) }\end{array}$ & $\begin{array}{l}\text { Cooke (2000), Bamber et al. (1999), Eti et al. (2004), } \\
\text { Davis (1997), Graisa y Al-Habaibeh (2011). }\end{array}$ \\
\hline
\end{tabular}

En lo que corresponde a los aspectos técnicos del MPT, se presentan en la Tabla 2. De acuerdo con la literatura revisada, un modelo para administrar con efectividad el mantenimiento requiere de un proceso en el cual las actividades relacionadas con la identificación, planeación, programación y la ejecución del trabajo se combinen correctamente, usando indicadores pertinentes que verifiquen el desempeño previsto. Este modelo incluye algunos de los 11 factores relacionados con aspectos técnicos del MPT y se agruparon en una categoría que incluye actividades inherentes a la aplicación de diversas herramientas de ingeniería de proceso y de mantenimiento, al uso de indicadores de desempeño, a la integración con herramientas de otras iniciativas de mejora continua, al uso de tecnologías de información y al establecimiento de una estructura organizacional específica para la implantación del MPT. 
Tabla 2.

Factores relacionados con aspectos técnicos.

\begin{tabular}{|c|c|}
\hline Factores identificados & Autores \\
\hline $\begin{array}{l}\text { Aplicación de un sistema computarizado } \\
\text { de administración del mantenimiento / } \\
\text { Tecnologías de información }\end{array}$ & $\begin{array}{l}\text { Bohoris et al. (1995), Brah y Chong (2004), Chand y } \\
\text { Shirvani (2000), Thun (2008). }\end{array}$ \\
\hline Mantenimiento descentralizado & Swanson (1997), Jonsson (1997). \\
\hline Rutinas de mantenimiento preventivo & $\begin{array}{l}\text { Ahuja y Kamba (2008c), Chinese y Ghirardo (2010), } \\
\text { Thomas et al. (2010). }\end{array}$ \\
\hline $\begin{array}{l}\text { Aplicar un enfoque de mejoramiento al } \\
\text { proceso / integración con TQM /Lean / JIT }\end{array}$ & $\begin{array}{l}\text { Ahuja y Kamba (2008c), Brah y Chong (2004), } \\
\text { Carannante et al. (1996), Al- Najjar (1996), Finlow- } \\
\text { Bates et al. (2000), Konecny y Thun (2011), Muthu et al. } \\
\text { (2001), Maletic et al. (2012). }\end{array}$ \\
\hline $\begin{array}{l}\text { Aplicar un programa de mantenimiento } \\
\text { centrado en la confiabilidad }\end{array}$ & $\begin{array}{l}\text { Ben-Daya (2000), Chan et al. (2005), Chinese y Ghirardo } \\
\text { (2010), Van der Wal y Lynn (2002) }\end{array}$ \\
\hline $\begin{array}{l}\text { Aplicación integral de herramientas de } \\
\text { Mantenimiento / Ingeniería }\end{array}$ & $\begin{array}{l}\text { Blanchard (1997), Carannante et al. (1996), Al- Najjar } \\
\text { (1996), Jonsson (1997), McAdam y McGeough (2000). }\end{array}$ \\
\hline $\begin{array}{l}\text { Uso y difusión de indicadores de } \\
\text { desempeño }\end{array}$ & $\begin{array}{l}\text { Carannante et al. (1996), Bamber et al. (1999), Ferrari et } \\
\text { al. (2002), Fredendall et al. (1997). }\end{array}$ \\
\hline $\begin{array}{l}\text { Distribución de planta para el } \\
\text { mantenimiento }\end{array}$ & Carannante et al. (1996). \\
\hline Desarrollo de una estructura propia & $\begin{array}{l}\text { Chan et al. (2005), Bamber et al. (1999), Jonsson (1997), } \\
\text { Kumar et al. (2006). }\end{array}$ \\
\hline Implementación de 5’S & Thomas et al. (2010), Gnanaguru et al. (2011). \\
\hline Uso de modelos matemáticos & Lawrence (1999), Wang y Lee (2001). \\
\hline
\end{tabular}

En lo referente a los aspectos relacionados con el desarrollo del recurso humano, Dess y Miller (1993) afirman que el capital humano es un factor importante y que su integración es vital para el despliegue exitoso de estrategias, pero también es pertinente destacar que en la práctica, la administración del recurso humano en las empresas no es una tarea sencilla: se desea que el personal trabaje unido, en armonía, con sinergia y en un clima de trabajo que impulse el desarrollo del personal y de su empresa. Sin embargo, es difícil que las organizaciones puedan conciliar efectivamente los intereses personales con los de la misma organización; nuevamente se presenta una tarea compleja para los líderes organizacionales, debido a que la forma de vida, en lo individual, es una característica personal de la idiosincrasia de la gente. En la Tabla 3 se presentan los factores del personal. 
Tabla 3.

Factores relacionados con el desarrollo del recurso humano.

\begin{tabular}{|c|c|}
\hline Factores identificados & Autores \\
\hline $\begin{array}{l}\text { Integración de todos los empleados / } \\
\text { trabajo en equipo / cooperación }\end{array}$ & $\begin{array}{l}\text { Bohoris et al. (1995), Chand y Shirvani (2000), Cooke } \\
\text { (2000), Bamber et al. (1999), Fredendall et al. (1997), } \\
\text { Jonsson (1997), Konecny y Thun (2011), Lycke (2003), } \\
\text { Park y Han (2001), Rolfsen y Langeland (2012). }\end{array}$ \\
\hline $\begin{array}{l}\text { Entrenamiento / } \\
\text { capacitación / educación }\end{array}$ & $\begin{array}{l}\text { Swanson (1997), Ahuja y Kamba (2008c), Carannante et } \\
\text { al. (1996), Chan et al. (2005), Eti et al. (2004), Ferrari et } \\
\text { al. (2002), Fredendall et al. (1997), Graisa y Al-Habaibeh } \\
\text { (2011), Konecny y Thun (2011), Lazim y Ramayah (2010), } \\
\text { Park y Han (2001). }\end{array}$ \\
\hline Involucramiento del empleado & $\begin{array}{l}\text { Ahuja y Kamba (2008c), Brah y Chong (2004), Cooke } \\
\text { (2000), Bamber et al. (1999), Fredendall et al. (1997), } \\
\text { Thomas et al. (2010), Parida y Kumar (2006). }\end{array}$ \\
\hline $\begin{array}{l}\text { Selección efectiva del equipo de } \\
\text { implementación }\end{array}$ & Chan et al. (2005), Lycke (2003). \\
\hline $\begin{array}{l}\text { Implementación de un sistema de } \\
\text { sugerencias }\end{array}$ & Konecny y Thun (2011), McAdam y McGeough (2000). \\
\hline
\end{tabular}

La Tabla 3 enlista los factores identificados en varias investigaciones o estudios de caso relacionados con esta categoría; incluye actividades vinculadas con la formación y desarrollo de equipos de trabajo, la capacitación y el entrenamiento pertinente, al igual que el involucramiento del empleado. Como se puede deducir de la revisión de la literatura, existen contradicciones implícitas entre los expertos, estas ambigüedades también manifiestan que, en estado actual, no es una teoría con el suficiente poder explicativo.

\section{Metodología}

La unidad de análisis está compuesta por gerentes, ingenieros, supervisores y personal técnico relacionado con el proceso de implantación del MPT provenientes de 65 plantas de la industria maquiladora que operan en Ciudad Juárez, México. La metodología se dividió en tres etapas y en los siguientes párrafos se describe cada una de ellas.

\section{Primera etapa: Diseño de la Encuesta}

Para la identificación de los FCE relacionados con la implantación del MPT y las posibles variables observables asociadas con dichos factores se aplicaron los procedimientos descritos por Hernández et al. (1998) y Lévy y Varela (2003). De los 31 factores identificados en las Tablas 1, 2 y 3 se extrajeron a priori ocho constructos y las variables que los miden, los cuales se ajustan con los resultados 
del Análisis Factorial; en el Anexo 1 se describe el nombre de las variables latentes y sus correspondientes indicadores. El éxito en la implantación del MPT se midió aplicando el enfoque de McKone et al. (2001), Cua et al. (2001) y Konecny y Thun (2011), quienes relacionaron la mejora percibida en el desempeño con el costo, la entrega, la calidad y la flexibilidad con algunas dimensiones del MPT. El cuestionario se dividió en nueve secciones, donde cada sección está relacionada con un constructo y se componen con ítems que se miden en una escala de Likert de 5 puntos, para que los participantes seleccionen su percepción en relación con la frecuencia en su empresa, con cada una de las actividades representadas en las afirmaciones en cada ítem (Lévy y Varela, 2003). Las preguntas de la 1 a la 74 son respondidas en una escala ordinal del 1 al 5, donde el 1 representa que nunca se observa la actividad y el 5 indica que siempre se observa tal actividad; esta escala fue utilizada en investigaciones similares (Ahuja y Kamba, 2008a; Konecny y Thun, 2011). La sección correspondiente al constructo desempeño del MPT está estructurada por cinco ítems, el primero de ellos mide —en una escala de Lickert de 5 puntos- el nivel de acuerdo del entrevistado en la reducción del tiempo muerto posterior a la implementación del MPT; las preguntas restantes se responden en una escala ordinal, donde el 1 representa un bajo nivel del desempeño operacional en relación con la competencia y un 5 representa un nivel superior de desempeño operacional en relación con la competencia (Konecny y Thun, 2011). Finalmente, se incluye una sección para recolectar información complementaria referente al giro y tamaño de la empresa; al sexo, antigüedad y puesto del participante y, de manera opcional, el nombre de la planta encuestada.

Para comprobar la validez de contenido del cuestionario (Lévy y Varela, 2003) se consultó la opinión de seis expertos en el tema de la implantación del MPT, para determinar que cada ítem tenga relación con el constructo que pretende medir; además, fueron consultados cinco expertos con experiencia en el diseño de cuestionarios, para verificar que la redacción de la pregunta reduzca el prejuicio de respuesta. Con objeto de validar la fiabilidad del instrumento, se calculó el índice de Alfa de Cronbrach en una muestra preliminar de 30 cuestionarios, seleccionando como punto de corte un valor de 0.70 (Hair et al., 2010).

\section{Segunda etapa: Aplicación del cuestionario a los participantes}

El cuestionario se aplicó en una muestra de la Industria Maquiladora de Exportación (IME) de Ciudad Juárez, a personal con conocimiento del proceso de implantación del MPT. El perfil de los puestos incluyó a ingenieros y supervisores de mantenimiento, ingenieros de diseño y de manufactura, ingenieros "lean", 
ingenieros de servicio al cliente, responsables de desarrollo de proveedores, supervisores de producción y de mantenimiento, entre otros, así como a gerentes de mantenimiento, de producción, de proyectos especiales, de calidad y, finalmente, gerentes de planta o corporativos. Para determinar el tamaño de muestra se utilizaron los criterios de Hair et al. (2010), que recomiendan al menos 100 casos para efectuar análisis estadísticos aplicando modelado con ecuaciones estructurales, y por MacCallum et al. (1999), quienes reportan que diversos estudios con muestras de al menos 200 casos que presentan comunalidades altas, ofrecen buenas soluciones factoriales. Así, se distribuyeron 400 cuestionarios, y se recolectaron 306.

\section{Tercera etapa: Identificación de los Factores Significativos (FCE)}

En esta etapa la información fue analizada con el paquete computacional SPSS 21. Se inició con un tratamiento inicial de los datos que incluyó pruebas de detección de datos extremos para identificar errores de captura, posteriormente se imputaron los datos ausentes aplicando el método de la Maximización de Valores Esperados, ya que en todas las variables observables, el porcentaje de valores perdidos fue menor a 10\% (Hair et al., 2010). Para evaluar la normalidad multivariada se calculó el índice de Mardia (Costello y Osborne, 2005) con AMOS 16. Con objeto de comprobar la adecuación de la muestra para el análisis factorial fueron aplicadas las pruebas de Kaiser-Meyer-Olkin y de esfericidad de Barlett, descritas en Lévy y Varela (2003). A fin de identificar los factores significativos, se eligió el método de factores de ejes principales (Costello y Osborne, 2005), tomando como criterio de extracción los componentes con Eigenvalores superiores a la unidad, contenidos en la matriz de correlaciones de las variables (ítems) analizadas. Con el propósito de mejorar la interpretación de la matriz de correlaciones fue aplicado el método de rotación Varimax, de Lévy y Varela (2003).

\section{Resultados}

Los resultados obtenidos se exponen en los siguientes apartados, iniciando con la descripción demográfica de la muestra, seguida de los resultados del tratamiento inicial a los datos y la validación estadística de fiabilidad del instrumento, finalmente se incluye el análisis factorial efectuado para la identificación de los FCE.

\section{Descripción demográfica de la muestra}

Se recolectaron 306 cuestionarios de empleados conocedores del MPT en una población de 65 plantas de la IME; como no todos los participantes respondieron de forma completa la sección relacionada con la información correspondiente al 
puesto, a la antiguiedad y al género, los resultados porcentuales relativos se presentan únicamente para el número de cuestionarios contestados. La información concentrada se muestra en la Tabla 4.

Tabla 4.

Perfil de los entrevistados.

\begin{tabular}{lll}
\hline Característica & Núm. de casos & Porcentaje \\
\hline Género & & \\
$\quad$ Masculino & 162 & $82 \%$ \\
$\quad$ Femenino & 36 & $18 \%$ \\
Puesto génerico ocupado & & \\
$\quad$ Gerente & 22 & $12 \%$ \\
$\quad$ Ingeniero de mantenimiento o área & 82 & $44 \%$ \\
$\quad$ Supervisor de mantenimiento o de producción & 59 & $31 \%$ \\
Administrativo & 6 & $3 \%$ \\
Jefe de grupo & 6 & $3 \%$ \\
Técnico en mantenimiento & 13 & $7 \%$ \\
Antigüedad en el puesto & & \\
Menos de 2 años & 39 & $19 \%$ \\
Entre 2 y 5 años & 66 & $35 \%$ \\
Entre 5 y 10 años & 59 & $31 \%$ \\
Más de 10 años & 29 & $15 \%$ \\
\hline
\end{tabular}

Fuente: Elaboración propia, a partir de los datos de la encuesta.

Como se puede observar, cerca de $90 \%$ de los entrevistados ocupan una posición jerárquica de mando, el restante $10 \%$ es personal operativo que ha participado en los programas de mejora continua en sus empresas, por lo cual se considera que la información obtenida es relevante para la investigación. Aproximadamente $46 \%$ de los participantes cuentan con una antigüedad mayor a 5 años, son empleados con experiencia y ocupan una posición de mando. La población de la IME es cercana a las 400 plantas (INEGI, 2009) y las empresas de la muestra son de los sectores automotriz, eléctrico, electrónico y médico, que en conjunto constituyen una fracción de $90 \%$ del empleo total de las plantas. Como se puede apreciar en la Tabla 5, 86\% de los encuestados pertenecen a los sectores industriales predominantes, mencionados con anterioridad.

Tabla 5 .

Distribución porcentual por sector industrial.

\begin{tabular}{lccccc}
\hline Sector Industrial & Automotriz & Eléctrica / Electrónica & Médico & Otros & Sin Referencia \\
\hline Distribución porcentual & $54 \%$ & $21 \%$ & $11 \%$ & $13 \%$ & $1 \%$ \\
\hline
\end{tabular}

Fuente: Elaboración propia, a partir de los datos de la encuesta. 
La muestra también se caracteriza con los empleados encuestados y el tamaño de las plantas donde trabajan: $92 \%$ de los encuestados son empleados de empresas que cuentan con más de 251 puestos de trabajo, $6 \%$ de los elementos de la muestra son de empresas medianas, con puestos de trabajo en el rango de 51 a 250 , y $2 \%$ son de empresas pequeñas y microindustrias con una planta menor de 50, y porque esta composición porcentual es representativa de la población. Esta integración también se explica porque se desea información sobre las empresas grandes como General Motors, Toyota, Mercedez Benz, Lexmark, Toshiba y Johnson \& Johnson, entre otras, que compiten intensamente en sus mercados y utilizan prácticas de clase mundial, como el MPT, para desarrollar sus competencias distintivas.

\section{Validación de la fiabilidad del Instrumento}

La validación de la consistencia interna de cada uno de los constructos identificados en la literatura se muestra en la Tabla 6.

Tabla 6.

Validación de la consistencia interna de las variables latentes.

\begin{tabular}{lccccccccc}
\hline & $\mathrm{A}$ & $\mathrm{B}$ & $\mathrm{C}$ & $\mathrm{D}$ & $\mathrm{E}$ & $\mathrm{F}$ & $\mathrm{G}$ & $\mathrm{H}$ & $\mathrm{I}$ \\
\hline Alfa de & 0.915 & 0.938 & .846 & .928 & .915 & .954 & .944 & .962 & .943 \\
Conbrach & 12 & 16 & & & & 52 & & & \\
Íltem a eliminar & $(.924)$ & $(.940)$ & - & - & - & $\begin{array}{c}(.955) \\
\text { I }\end{array}$ & - & - & - \\
\hline
\end{tabular}

A: Proceso Planeación Estratégica; B: Desarrollo del Recurso Humano; C: Distribución de planta para el MPT; D: Contribuciones Gerenciales; E: Comunicación del Proceso de Implementación; F: Técnicas Básicas del MPT; G: Prácticas de Mejora Continua; H: Estructura Propia; I: Desempeño de la empresa posterior a la implantación del MPT.

Fuente: Elaboración propia, a partir de los resultados de la encuesta.

Los valores del Alfa de Cronbach muestran, en todos los constructos, una alta fiabilidad del instrumento, ya que superan el valor mínimo recomendado de 0.75 (Lévy y Varela, 2003; Hair et al., 2010). Los valores en paréntesis indican el valor $\alpha$ si se elimina el ítem especificado, sin embargo no se omitieron debido a las altas correlaciones con el resto de la variables ( $\mathrm{r}$ corregida $>0.30$ ).

\section{Comprobación de la adecuación de la muestra}

Las pruebas de adecuación muestral de Kaiser-Meyer-Olkin y de esfericidad de Barlett fueron efectuadas con el paquete estadístico SPSS 21, la Tabla 7 muestra los valores calculados en los 306 cuestionarios. 
Tabla 7.

Prueba de KMO y de esfericidad de Barlett.

\begin{tabular}{lll}
\hline Medida de adecuación muestral de Kaiser-Meyer-Olkin. & .966 \\
\hline Prueba de esfericidad de & Chi-cuadrado aproximado & 22016.763 \\
& Gl & 3081 \\
Bartlett & Sig. & .000 \\
\hline
\end{tabular}

Fuente: Elaboración propia, a partir de los resultados de la encuesta.

El valor obtenido de la prueba KMO es de 0.966 , mayor al mínimo de 0.80 recomendado por Lévy y Varela (2003), es decir, las correlaciones parciales son pequeñas y, por lo mismo, están midiendo un mismo factor. Respecto a la prueba de esfericidad de Barlett, la significancia es cero, por ello se rechaza la hipótesis de que la matriz de correlaciones es una matriz identidad, esto implica que hay una proporción muy alta de correlaciones significativas entre las variables, pudiéndose agrupar en componentes significativos.

\section{Análisis Factorial}

En este apartado se presentan los resultados obtenidos de la aplicación del Análisis Factorial de los datos recolectados para ajustar los FCE identificados en la revisión de la literatura, disminuyendo el número de las variables que miden cada uno estos factores.

Tabla 8.

Varianza explicada por los factores.

\begin{tabular}{|c|c|c|c|c|c|c|}
\hline \multirow[b]{2}{*}{ Núm. } & \multicolumn{3}{|c|}{ Eigen valor inicial } & \multicolumn{3}{|c|}{ Eigen valor rotado } \\
\hline & Total & $\begin{array}{l}\% \\
\text { Varianza }\end{array}$ & $\begin{array}{l}\% \\
\text { Acumulado }\end{array}$ & Total & $\begin{array}{l}\% \\
\text { Varianza }\end{array}$ & $\begin{array}{l}\% \\
\text { Acumulado }\end{array}$ \\
\hline 1 & 37.863 & 47.928 & 47.928 & 9.004 & 11.398 & 11.398 \\
\hline 2 & 3.016 & 3.818 & 51.747 & 6.501 & 8.230 & 19.627 \\
\hline 3 & 2.594 & 3.284 & 55.030 & 5.323 & 6.738 & 26.366 \\
\hline 4 & 2.005 & 2.538 & 57.569 & 5.136 & 6.501 & 32.867 \\
\hline 5 & 1.670 & 2.113 & 59.682 & 5.087 & 6.439 & 39.306 \\
\hline 6 & 1.598 & 2.023 & 61.705 & 4.202 & 5.319 & 44.625 \\
\hline 7 & 1.505 & 1.906 & 63.610 & 4.041 & 5.115 & 49.740 \\
\hline 8 & 1.333 & 1.688 & 65.298 & 3.417 & 4.325 & 54.066 \\
\hline 9 & 1.244 & 1.574 & 66.872 & 3.259 & 4.126 & 58.191 \\
\hline 10 & 1.173 & 1.484 & 68.357 & 3.231 & 4.090 & 62.281 \\
\hline 11 & 1.097 & 1.388 & 69.745 & 1.960 & 2.482 & 64.762 \\
\hline 12 & 1.046 & 1.324 & 71.069 & .879 & 1.113 & 65.875 \\
\hline
\end{tabular}

Fuente: Elaboración propia, a partir de los resultados de la encuesta. 
El valor del índice de Mardia de la kurtosis multivariante al conjunto de los 79 indicadores que conforman el cuestionario fue de 239.207, con un valor estandarizado de 36.997, lo que indica una desviación significativa de la normalidad multivariante; debido a ello, los factores significativos se extrajeron con el método de factorización de ejes principales (Costello y Osborne, 2005), y se aplicó el criterio de Kaiser (Hair et al., 2010) para determinar el número de factores a retener, tomando como base la matriz de correlaciones. Asimismo, fue aplicado el método de rotación Varimax para mejorar la interpretación de la solución factorial, tomando como criterio de retención saturaciones mayores a 0.5 en los factores extraídos por las variables analizadas. Los resultados de estos procedimientos se ilustran en la Tabla 8 y, como se puede apreciar, son 12 componentes los que explican aproximadamente el $71 \%$ de la varianza, sin embargo los factores 11 y 12 presentan saturaciones muy bajas, por lo que carecen de una interpretación significativa; así, se consideraron solo 10 factores que explican $68.4 \%$ de la varianza total de los datos. Los resultados de la matriz rotada, en la cual se ilustra el ítem y la carga factorial asociada, aparecen en la Tabla 9; los valores resaltados en letra itálica representan la carga factorial del ítem en el factor correspondiente.

Tabla 9.

Resultados de la matriz rotada.

\begin{tabular}{|c|c|c|c|c|c|c|c|c|c|c|}
\hline \multirow{2}{*}{ Factor } & \multicolumn{10}{|c|}{ Indicadores relacionados / carga factorial } \\
\hline & I6 & I8 & I4 & I7 & I9 & I5 & I3 & I2 & I1 & I11 \\
\hline 1 & 0.689 & 0.670 & 0.658 & 0.656 & 0.654 & 0.652 & 0.649 & 0.630 & 0.610 & 0.542 \\
\hline \multirow{2}{*}{2} & I68 & I66 & I64 & I69 & I65 & I67 & I71 & I70 & I73 & \\
\hline & 0.604 & 0.602 & 0.565 & 0.558 & 0.556 & 0.530 & 0.525 & 0.517 & 0.503 & \\
\hline \multirow{2}{*}{3} & I77 & I78 & I79 & I76 & I75 & & & & & \\
\hline & 0.789 & 0.774 & 0.701 & 0.688 & 0.620 & - & - & - & - & - \\
\hline \multirow{2}{*}{4} & I29 & $\mathrm{I} 30$ & - & - & - & - & - & - & - & - \\
\hline & 0.522 & 0.567 & - & - & - & - & - & & & - \\
\hline \multirow{2}{*}{5} & I47 & I48 & I49 & & & & & & & \\
\hline & 0.608 & 0.682 & 0.679 & - & - & - & - & - & - & - \\
\hline \multirow{2}{*}{6} & I15 & I16 & & & & & & & & \\
\hline & 0.606 & 0.737 & - & - & - & - & - & - & - & - \\
\hline \multirow{2}{*}{7} & I59 & I60 & I61 & I62 & I63 & & & & & \\
\hline & 0.742 & 0.655 & 0.604 & 0,546 & 0.503 & - & - & - & - & - \\
\hline \multirow{2}{*}{8} & I40 & I41 & I42 & & & & & & & \\
\hline & 0.602 & 0.617 & 0.502 & - & - & - & - & - & - & - \\
\hline \multirow{2}{*}{9} & I51 & I53 & I54 & - & & & & & & \\
\hline & 0.529 & 0.665 & 0.678 & - & - & - & - & - & - & - \\
\hline \multirow{2}{*}{10} & $\mathrm{I} 21$ & $\mathrm{I} 22$ & $\mathrm{I} 23$ & & & & & & & \\
\hline & 0.569 & 0.557 & 0.583 & - & - & - & - & - & - & - \\
\hline
\end{tabular}

Fuente: Elaboración propia, a partir de los resultados de la encuesta. 
Como se puede apreciar en la Tabla 9, los factores 1,4 y 8 coinciden con los constructos identificados a priori como Proceso de Planeación Estratégica, Distribución de planta para el MPT y Comunicación del Proceso de Implementación, respectivamente, y representan FCE. Asimismo,, el Análisis Factorial coincide con la identificación a priori del constructo Desempeño de la empresa posterior a la implantación del MPT, como una variable latente que caracteriza el éxito en la implantación del MPT, el cual es representado por el factor 3. El factor 2 combina indicadores relacionados con la Aplicación de herramientas de mejora continua propias de la Administración total de la calidad, el Justo a Tiempo y algunas actividades relacionadas con la capacitación y difusión específica del MPT, a este factor se le denominó Integración del MPT con prácticas de mejora continua. Inicialmente se propuso que el constructo Técnicas Básicas del MPT integrara actividades relacionadas con el mantenimiento preventivo, la incorporación de tecnología de vanguardia al equipo de producción, la innovación para el desarrollo de nuevo equipo o la adaptación del existente, y la aplicación de un programa de 5's. Sin embargo, el Análisis Factorial separó este constructo en dos factores relevantes: el actor 5, compuesto por actividades relacionadas con la incorporación de tecnología de vanguardia al equipo de producción, y el actor 9, vinculado con la innovación para el desarrollo de nuevo equipo, o para la adaptación del ya existente.

De igual manera, los factores 6 y 10 se componen de indicadores que previamente se habían agrupado en el constructo Desarrollo del Recurso Humano, pero se separaron en dos factores distintos, que son el Mantenimiento Autónomo y el Desarrollo de Equipos de Trabajo MPT. El primero de ellos incluye actividades enfocadas en que los operadores de producción efectúen tareas relacionadas con rutinas de mantenimiento preventivo; el segundo está conformado por acciones encaminadas al desarrollo de equipos de trabajo específicos para el despliegue y seguimiento del proceso de implantación del MPT. Finalmente, al actor 7 se le denominó Integración con Clientes y Proveedores, y se compone de actividades enfocadas a vincular las necesidades de los clientes con la función del mantenimiento y el involucramiento de los proveedores en las tareas de diseño de equipo para la producción. Estos resultados muestran una adecuada consistencia con los FCE identificados en la literatura.

Para concluir con el análisis de los resultados, se inspeccionaron los residuales no redundantes entre las matrices de correlaciones observadas y reproducidas, encontrándose que $3 \%$ de ellos tienen valores absolutos mayores a 0.05 , con lo cual se comprueba la calidad de la solución factorial propuesta en este estudio. 


\section{Conclusiones}

En la revisión de literatura se identificaron 31 factores relacionados con la implantación del MPT, los cuales sirvieron de base para diseñar un cuestionario y discriminarlos por su impacto en empresas manufactureras de la industria maquiladora en Ciudad Juárez. Con la aplicación del Analisis Factorial se identificaron 10 constructos, nueve de ellos representan FCE y uno mide la mejora en el desempeño operacional posterior a la implantación del MPT. Los FCE identificados incluyen 40 actividades clave, que fueron agrupadas en las siguientes variables latentes:

Alineación estratégica del MPT. Esta orientación implica incorporar en la planeación estratégica de la compañía políticas explícitas para desarrollar a largo plazo el MPT, lo cual conlleva el contar con un plan de implantación propio que contenga una declaración de misión y propósitos, indicadores de desempeño, metas, tiempos de implantación, responsabilidades y un plan de reconocimientos del desempeño, para reducir la resistencia al cambio.

Integración con prácticas de mejora continua. Este factor implica la integración de herramientas contenidas en la Administración Total de la Calidad y el Justo a Tiempo como un marco de referencia que potencia la implantación del MPT. En este factor se incluyen las actividades requeridas para la capacitación y sensibilización del MPT, lo cual explica porqué estos aspectos son comunes para el despliegue del JAT y la ATC.

Distribución de planta para el MPT. En esta categoría se resaltan, por un lado, la importancia que tiene el diseño de las instalaciones para la adecuada capacidad de operación del equipo de producción y, por otro, que la distribución de la maquinaria tome en cuenta accesos, para que el mantenimiento sea fácil de efectuar.

Mantenimiento Autónomo. Es uno de los principales pilares del MPT y se distingue como un FCE; implica asignarle al operario la responsabilidad del mantenimiento preventivo de la maquinaria asignada, previa capacitación.

Alineación del equipo de trabajo MPT. Este FCE se compone de acciones encaminadas a conformar un equipo de trabajo multidisciplinario y enfocado al despliegue del MPT, cuyos integrantes reúnan atributos de liderazgo y sus metas estén alineadas con el plan estratégico de implantación.

Integración con Clientes y Proveedores. Esta integración permite, para su despliegue, estrechar relaciones con los clientes a fin fr identificar sus necesidades, que deben ser consideradas durante el diseño del equipo, de esta forma las rutinas del mantenimiento consideran los requisitos de cliente en cuanto a calidad y tiempo de entrega. Además, se debe considerar mantener estrechas relaciones con los proveedores del equipo de producción y con quienes suministran las refacciones, prefiriendo los certificados. 
Aplicación de Tecnología de Vanguardia. En el contexto estudiado, las empresas que se enfocan a lograr el liderazgo en la incorporación de tecnología de vanguardia implantan efectivamente el MPT, dado que la renovación del equipo de producción ofrece la oportunidad de adquirirlo con mejor nivel de mantenibilidad.

Comunicación del Proceso de Implantación. La resistencia al cambio es una de las principales barreras al momento de desplegar la estrategia. Para el caso del MPT, se recomienda comunicar con objetividad los beneficios obtenidos durante el proceso de implantación, pues es un factor clave, ya que los departamentos responsables de su ejecución adoptarán los cambios requeridos por el MPT.

Desarrollo de Equipo Propio. Es necesario que la invención y el mejoramiento del equipo de producción tomen en cuenta los requisitos de mantenibilidad, de tal forma que se desarrollen paquetes tecnológicos dificiles de imitar por los competidores, lo cual representa un factor clave para la implantación exitosa del MPT.

Los FCE determinados constituyen la principal contribución de esta investigación a la teoría del campo de los sistemas administrativos, se observan coincidencias con algunos de los factores críticos que se mencionan en la literatura, aunque también se descartan como FCE otros factores allí citados, que no parecen ser críticos. Además, los resultados de esta investigación constituyen evidencia de que el despliegue efectivo del MPT forma parte de una estrategia de manufactura enfocada en la creación y desarrollo de competencias de manufactura, para obtener una ventaja competitiva sostenible. Asimismo, se considera que los factores expuestos constituyen un marco de referencia que le puede permitir a los directivos de las empresas administrar las actividades clave predictoras de la implantación exitosa del MPT. Es recomendable replicar este tipo de proyectos para enriquecer e incrementar la evidencia, y probar la generalidad de los FCE propuestos. 


\section{Anexo}

\section{Cuestionario}

\begin{tabular}{|c|c|}
\hline \multicolumn{2}{|l|}{ Constructo: Proceso de planeación estratégica } \\
\hline Variables observables & Código \\
\hline $\begin{array}{l}\text { Las responsabilidades de nuestro programa de mantenimiento estén bien establecidas para todas las } \\
\text { áreas de la planta. }\end{array}$ & I1 \\
\hline $\begin{array}{l}\text { La formulación de la misión, estrategias y políticas de la empresa reflejen el compromiso de la alta } \\
\text { gerencia con el mantenimiento del equipo. }\end{array}$ & $\mathrm{I} 2$ \\
\hline Nuestras metas corporativas fomenten y apoyen el desarrollo de un programa de mantenimiento. & I3 \\
\hline La declaración de la misión sea comunicada efectivamente en toda la empresa. & I4 \\
\hline La declaración de la misión sea completamente apoyada por nuestros empleados. & I5 \\
\hline El plan de implementación considere una política para el mantenimiento planeado. & I6 \\
\hline $\begin{array}{l}\text { El plan de implementación considera una política para incorporar prácticas de mantenimiento } \\
\text { predictivo. }\end{array}$ & I7 \\
\hline $\begin{array}{l}\text { El plan de implementación considere indicadores para evaluar el desempeño del programa de } \\
\text { mantenimiento. }\end{array}$ & I8 \\
\hline El plan de implementación prevea la introducción gradual del programa. & I9 \\
\hline La asignación de recursos financieros para apoyar la implementación sea suficiente. & I10 \\
\hline El plan prevea un horizonte de largo plazo para implementar completamente el programa. & I11 \\
\hline El plan establezca un sistema de incentivos para premiar los logros obtenidos. & I12 \\
\hline \multicolumn{2}{|l|}{ Constructo: Desarrollo del recurso humano } \\
\hline Variables observables & Código \\
\hline
\end{tabular}

Se formen equipos para resolver problemas de producción y fomentar la participación de los empleados.

Los empleados dispongan de los recursos suficientes para tratar con problemas de paros de equipo inesperados.

A los operadores se les haya dado entrenamiento en principios de mantenimiento autónomo.

Los operadores sean responsables del mantenimiento de sus máquinas.

Los operadores sean responsables de inspeccionar su propio trabajo.

Exista un programa que garantice que las sugerencias de los empleados sean regularmente evaluadas e implementadas.

Exista un programa que garantice que la administración comunique por qué las sugerencias fueron o no implementadas.

La habilidad para resolver problemas sea un criterio de promoción del empleado.

Los equipos de MPT están enfocados de acuerdo con metas preestablecidas.

Las metas de los equipos de MPT estén alineadas con las metas de la empresa.

Los responsables de los equipos de MPT cuenten con experiencia en el sistema y tengan cualidades de liderazgo.

Los empleados de los diferentes departamentos de la planta cooperen entre sí para resolver sus conflictos.

Los empleados de los departamentos administrativos conozcan los problemas de producción.

Los empleados reciban entrenamiento para desempeñar múltiples tareas.

Los empleados reciban entrenamiento cruzado, para que puedan sustituir a otros si es necesario. 


\begin{tabular}{|c|c|}
\hline \multicolumn{2}{|l|}{ Constructo: Distribución de planta para el MPT } \\
\hline Variables observables & Código \\
\hline $\begin{array}{l}\text { Las instalaciones de suministro de energía (electricidad, fluidos, gas, etc.) se diseñen con las } \\
\text { capacidades de operación requeridas por los equipos de producción. }\end{array}$ & I 29 \\
\hline La distribución del equipo manufactura tome en cuenta accesos para el fácil mantenimiento. & $\mathrm{I} 30$ \\
\hline Constructo: Contribuciones gerenciales & \\
\hline Variables observables & Código \\
\hline Se perciba un liderazgo de la dirección en la ejecución de los programas de MPT. & I31 \\
\hline La dirección cree y comunique una visión centrada en calidad y mantenimiento. & $\mathrm{I} 32$ \\
\hline Los jefes de departamento acepten su responsabilidad hacia el MPT. & $\mathrm{I} 33$ \\
\hline Existan reuniones de trabajo entre el departamento de mantenimiento y producción. & $\mathrm{I} 34$ \\
\hline $\begin{array}{l}\text { La gerencia promueva la participación de trabajadores en el mantenimiento y conservación del } \\
\text { equipo. }\end{array}$ & $\mathrm{I} 35$ \\
\hline La dirección se involucre en proyectos para mejorar los resultados del programa de mantenimiento. & I36 \\
\hline La dirección defina e identifique indicadores para el desempeño del programa de mantenimiento. & $\mathrm{I} 37$ \\
\hline La dirección promueva activamente una cultura de MPT. & $\mathrm{I} 38$ \\
\hline La dirección pondera correctamente el MPT y el programa de producción. & I39 \\
\hline
\end{tabular}

\section{Constructo: Comunicación del proceso de implantación}

Variables observables

Código

La frecuencia y duración del tiempo muerto debido a paros, set-up, microparos, reducción de

velocidad, defectos, retrabajo y pérdidas del rendimiento sean graficados y mostrados en el piso de producción.

Empleados tengan fácil acceso a la información de la productividad.

La dirección promueva sesiones de sensibilización previas a cualquier cambio radical en los sistemas de manufactura.

Constructo: Técnicas básicas del MPT

Variables observables

Código

Los operarios del equipo dediquen una porción de cada día solamente al mantenimiento.

Se considere al mantenimiento como una estrategia para cumplir con los requerimientos de entrega y calidad del cliente.

Nosotros tengamos un turno, o parte del mismo, dedicado a actividades de mantenimiento.

Nuestro departamento de mantenimiento se enfoque en asistir a los operarios para desempeñar su propio mantenimiento preventivo.

Nuestra planta se mantenga a la vanguardia en las nuevas tecnologías en nuestra industria.

En nuestra planta estemos constantemente pensando en la siguiente generación de tecnología.

Nuestra planta sea líder en el uso efectivo de nuevas tecnologías para procesos de manufactura.

Los ingenieros de la planta busquen permanentemente aprender y mejorar el equipo después de su instalación.

Nosotros desarrollemos equipo propio y específico para nuestros procesos.

Contemos con proveedores para la mayoría de nuestros equipos.

Contemos con equipo propio que esté protegido por patentes de la firma.

El desarrollo de equipo propio nos ayude a obtener una ventaja competitiva. $\quad$ I54

El programa garantice que los operarios mantengan sus áreas de trabajos limpios y ordenados. I55

La planta se distinga por estar limpia en todo momento y en todos los departamentos. 
Los operarios tengan la disciplina de poner todas las herramientas y accesorios en su lugar.

La alta gerencia sea ejemplo del orden y limpieza en su área de trabajo.

Constructo: Prácticas de mejora continua

Variables observables

Nosotros estemos en estrecho contacto con nuestros clientes.

Código

Nos esforzemos por ser sensibles a las necesidades de nuestros clientes.

I59

Nos esforzemos por establecer relaciones de largo plazo con nuestros proveedores.

Contemos con un número pequeño de proveedores, pero de alta calidad.

I61

Nuestros proveedores cuenten con certificaciones que acrediten su calidad.

Nosotros monitoreemos nuestros procesos, aplicando técnicas de control estadístico.

Un gran porcentaje de nuestros equipos o procesos estén bajo control estadístico de calidad.

En la empresa se reducen continuamente los tiempos de set-up.

Los tiempos de ciclo se reduzcan continuamente.

El sistema de control de producción se base en un sistema de jalón (pull system).

En la planta se utilice el control visual para el piso de producción.

I68

I69

Constructo: Estructura propia

Variables observables

Código

En nuestra planta se desarrolle un plan maestro de capacitación para cada nivel jerárquico de los trabajadores.

El programa de capacitación se actualice continuamente.

En la planta se dé seguimiento permanente a las lecciones aprendidas del MPT.

En la planta se promueva continuamente la filosofía del MPT.

En la planta se difundan las buenas o malas experiencias obtenidas durante la implementación del MPT.

Constructo: Desempeño de la empresa

Variables observables

Código

La implementación del MPT en las diferentes líneas de producción ha generado una reducción en el

tiempo muerto por paros en el equipo.

Comparado con nuestros competidores, nuestro desempeño es mejor en costo unitario de manufactura.

Comparado con nuestros competidores, nuestro desempeño es mejor en calidad del producto.

Comparado con nuestros competidores, nuestro desempeño es mejor en tiempo y confiabilidad de entrega del producto.

Comparado con nuestros competidores, nuestro desempeño es mejor en flexibilidad para cambiar la mezcla y volumen del producto.

\section{Referencias}

Ahmed Shamsuddin, Hassan Masjuki Hj. and Taha Zahari (2005). "TPM can go beyond maintenance: excerpt from a case implementation", Journal of Quality in Maintenance Engineering, $11(1), 19-42$.

Ahuja I.P.S. and Khamba J.S. (2007). "An evaluation of TPM implementation initiatives in an Indian manufacturing enterprise", Journal of Quality in Maintenance Engineering, 13 (4), 338-352.

Ahuja I.P.S. and Khamba J.S. (2008a). "Assessment of contributions of successful TPM initiatives towards competitive manufacturing”, Journal of Quality in Maintenance Engineering, 14 (4), 356-374. 
Ahuja I.P.S. and Khamba J.S. (2008b). "Total productive maintenance: literature review and directions", International Journal of Quality \& Reliability Management, 25 (7), 709-756.

Ahuja I.P.S. and Khamba J.S. (2008c). "Strategies and success factors for overcoming challenges in TPM implementation in Indian manufacturing industry", Journal of Quality in Maintenance Engineering, 14, (2), 123-147.

Al-Hassan Khalid, Joseph Fat-Lam Chan and Andrew Viggo Metcalfe (2000). "The role of total productive maintenance in business excellence", Total Quality Management, 11 (4-6), 596-601.

Al-Najjar, Basim (1996). "Total quality maintenance. An approach for continuous reduction in costs of quality products”, Journal of Quality in Maintenance Engineering, 2 (3), 4-20.

Alsyouf, I. (2006). "Measuring maintenance performance using a balanced scorecard approach", Journal of Quality in Maintenance Engineering, 12, (2), 133-49.

Avella Lucia, Esteban Fernández y Camilo J. Vázquez (2001). “Analysis of manufacturing strategy as an explanatory factor of competitiveness in the large Spanish industrial firm", International Journal of Production Economics, 7 (2), 139-157.

Bamber, C.J., J.M. Sharp and M. Hides (1999). "Factors affecting successful implementation of total productive maintenance: a UK manufacturing case study perspective", Journal of Quality in Maintenance Engineering, 5 (3), 162-81.

Ben-Daya, Mohamed (2000). "You may need RCM to enhance TPM implementation", Journal of Quality in Maintenance Engineering, 6 (2), 82-85.

Blanchard, Benjamin S. (1997). "An enhanced approach for implementing total productive maintenance in the manufacturing environment", Journal of Quality in Maintenance Engineering, 3 (2), 69-80.

Bohoris, G.A., C. Vamvalis, W Tracey and K. Ignatiadou (1995). “TPM implementation in Land-Rover with the assistance of a CMMS", Journal of Quality in Maintenance Engineering, 1 (4), 3-16.

Brah, S.A. and W.K. Chong (2004). "Relationship between total productive maintenance and performance", International Journal of Production Research, 42 (12), 2383-2401.

Bruun, Peter and Robert N. Mefford (2004). "Lean Production and Internet", International Journal of Production Economics, 89 (3), 247-260.

Bunge, Mario (2004). La investigación científica. Primera Edición. Siglo XxI Editores, México, D.F.

Caralli, Richard A. (2004). "The Critical Success Factor Method: Establishing a Foundation for Enterprise Security Management”, Technical Report CMU/SEI-2004-TR-010 ESC-TR 2004-010, $1-135$.

Carannante, T., R.H. Haigh and D.S. Morris (1996). "Implementing total productive maintenance: A comparative study of the UK and Japanese foundry industries", Total Quality Management, 7 (6), 605- 611.

Chan, F.T.S., H.C.W. Lau, R.W.L. Ip, H.K. Chan and S- Kong (2005). "Implementation of Total Productive Maintenance: A case study", International Journal of Production Economics, 95 (1), 71-94.

Chand, G. and B. Shirvani (2000). "Implementation of TPM in cellular manufacture", Journal of Materials Processing Technology (103), 149-154.

Chinese, D. and G. Ghirardo (2010). "Maintenance management in Italian manufacturing firms: Matters of size and matters of strategy", Journal of Quality in Maintenance Engineering, 16 (2), 156-180.

Chen, Lixia and Bo Meng (2011). "The Three-stage method for chinese enterprises to deploy TPM", Management Science and Engineering, 5 (1), 51-58. 
Cooke, Fang Lee. (2000). "Implementing TPM in plant maintenance: some organisational barriers", International Journal of Quality \& Reliability Management, 17 (9), 1003-1016.

Costello, Anna B. and Jason W. Osborne (2005). "Best Practices in Exploratory Factor Analysis: Four Recommendations for Getting the Most from Your Analysis", Practical Assessment, Research \& Evaluation, 10 (7), 1-9.

Cua, Kisty O., Kathleen E. McKone and Roger G. Schroeder (2001). "Relationships between implementation of TQM, JIT, and TPM and manufacturing performance", Journal of Operations Management, 19, 675-694.

Dangayach, G. S. and S.G. Deshmukh (2006). "Implementation of manufacturing strategy: a select study of Indian process companies", Production Planning \& Control, 12 (1), 89-105.

Davis, R. (1997). "Making TPM a part of factory life", TPM Experience (Project EU 1190, DTI, Findlay, sponsored by the DTI.

Dess Gregory G. and Alex Miller (1993). Strategic Management. McGraw-Hill, USA.

Eti, M.C., S.O.T. Ogaji and S.D. Probert (2004). "Implementing total productive maintenance in Nigerian manufacturing industries", Applied Energy, 79, 385-401.

Ferrari, E., A. Pareschi, A. Persona and A. Regattieri (2002). "TPM: situation and procedure for a soft introduction in Italian factories", The TQM Magazine, 14 (6), 350-8.

Finlow-Bates, T., B. Visser B. and C. Finlow-Bates (2000). "An integrated approach to problem solving: linking K-T, TQM and RCA to TPM", The TQM Magazine, 12 (4), 284-289.

Fredendall, L.D., J.W. Patterson, W.J. Kennedy and T. Griffin (1997). "Maintenance modeling, its strategic impact", Journal of Managerial Issues, 9 (4), 440-53.

García, J.L., J. Romero y M.S. Noriega (2012). "El Éxito del Mantenimiento Productivo Total y su Relación con los Factores Administrativos". Revista Contaduría y Administración, 57 (4), 173-196.

Garg, Amik and S.G. Deshmukh (2006). "Maintenance management: literature review and directions", Journal of Quality in Maintenance Engineering, 12 (3), 205-238.

Gnanaguru, R., K. Puvaneswari and J. Mallick (2011). "Toyota's A3 reports for improving 6-S activities: an aeronautical industry case study", International Journal of Services and Operations Management, 10 (2), 239-254.

Graisa, Mustafa and Amin Al-Habaibeh (2011). "An investigation into current production challenges facing the Libyan cement industry and the need for innovative Total Productive Maintenance (TPM) strategy", Journal of Manufacturing Technology Management, 22 (4), 541-558.

Gyampah A. K. and Moses Acquaah (2008). "Manufacturing strategy, competitive strategy and firm performance: An empirical study in a developing economy environment", International Journal of Production Economics, 111 (2), 575-592.

Hair Jr., Joseph F., C. Black Williams, Barry J. Babin and Rolph E. Anderson Rolph (2010). Multivariate Data Analysis. Seventh Edition. Cornell University, Pearson Prentice Hall.

Hansson, J. and F. Backlund (2003). "Managing commitment: increasing the odds for successful implementation of TQM, TPM or RCM", International Journal of Quality \& Reliability Management, 20 (9), 993-1008.

Hayes, Robert H. and Steven C. Wheelwright (1984). Restoring Our Competitive Edge: Competing Through Manufacturing. John Wiley \& Sons Ltd.

Hernández Sampieri R., C. Fernández Collado y P. Baptista Lucio. (1998). Metodología de la investigación. Segunda Ed. México, D.F. 
Houshmand, Mahmound and Bizhan Jamsshidnezhad (2006). "An extended model of design process of lean production system by mean of process variables", Robotics and Computer-Integrated Manufacturing, 22, 1-16.

Jonsson, P. (1997). "The status of maintenance management in Swedish manufacturing firms", Journal of Quality in Maintenance Engineering, 3 (4), 233-58.

Konecny, Philipp A. and Jorn-Henrik Thun (2011). "Do it separately or simultaneously-An empirical analysis of a conjoint implementation of TQM and TPM on plant performance", International Journal of Production Economics, 133 (2), 496-504.

Kumar, S.R., D. Kumar D. and P. Kumar (2006). "Manufacturing excellence through TPM implementation: a practical analysis", Industrial Management \& Data Systems, 106 (2), 256-280.

Lawrence, J.L. (1999). "Use mathematical modeling to give your TPM implementation effort an extra boost", Journal of Quality in Maintenance Engineering, 5 (1), 62-9.

Lazim, H.M. and T. Ramayah (2010). "Maintenance strategy in Malaysian manufacturing companies: a total productive maintenance (TPM) approach", Business Strategy Series, 11 (6), 387396.

Leidecker, J.K. and A.V. Bruno (1984). "Identifying and Using Critical Success Factors". Long Range Planning (UK), 17 (1), 23-32.

Lévy Mangin, Jean-Pierre y Jesús Varela Mallou (2003). Análisis Multivariable para las Ciencias Sociales. Primera Edicion. Madrid: Pearson Educación.

Lycke, L. (2003). "Team development when implementing TPM", Total Quality Management, 14 (2), 205-213.

Maletic, Damjan, Matjaz Maletic and Bostjan Gomiscek (2012). "The relationship between continuous improvement and maintenance performance", Journal of Quality in Maintenance Engineering, 18 (1), 30-41.

Martínez-Berumen, Héctor A. and Carlos E. Escobar-Toledo (2011). "Decision-Making in new technologies for public research centers: a methodological proposal with systems approach", Systems Research Forum, 5, 53-72.

MacCallum, R.C., K.F. Widaman, S.B. Zhang and S.H. Hong (1999). "Sample size in factor analysis", Psychological Methods, 4 (1), 84-99.

McAdam, R. and F. McGeough (2000). "Implementing total productive maintenance in multi-union manufacturing organization: overcoming job demarcation”, Total Quality Management, 11 (2), 187-197.

McKone, Kathleen E., Roger G. Schroeder and Kristy O. Cua (2001). "The impact of total productive maintenance practices on manufacturing performance", Journal of Operations Management $19,39-58$

Muchiri, Peter, Liliane Pintelon, Ludo Gelders and Harry Martin (2011). "Development of maintenance function performance measurement framework and indicators", International Journal of Production Economics, 131 (1), 295-302.

Muthu, S., S.R. Devadasan, P.S. Mendonca and G. Sundararaj (2001). "Pre-auditing through a knowledge base system for successful implementation of a QS 9000 based maintenance quality system", Journal of Quality in Maintenance Engineering, 7 (2), 90-104.

Nakajima, S. (1988). Introduction to TPM: Total Productive Management. Portland, OR: Productivity Press.

Park, K.S. and S.W. Han (2001). "TPM-Total Productive Maintenance: Impact on Competitiveness and a Framework for Successful Implementation", Human Factors and Ergonomics in Manufacturing, 11 (4), 321-338. 
Parida, A. and U. Kumar (2006). "Maintenance Performance Measurement (MPM): issues and challenges", Journal of Quality in Maintenance Engineering, 12 (3), 239-251.

Pintelon, Liliane, Kumar Pinjala Srinivas and Ann Vereecke (2006). "Evaluating the effectiveness of maintenance strategies", Journal of Quality in Maintenance Engineering, 12 (1), 7-20.

Plant R. and L. Willcocks (2007). "Critical success factors in international ERP implementations: A case research approach", Journal of Computer Information Systems, 47 (3), 60.

Rho Boo-Ho, Kwangtae Park and Yung-Mok Yu (2001). "An international comparison of the effect of manufacturing strategy-implementation gap on business performance", International Journal of Production Economics 70 (1), 89-97.

Rockart, J.F. and C.V. Bullen (1981). "A Primer on Critical Success Factors," (Center for Information Center Research. Sloan School of Management. M.I.T. Working Paper No. 69, June).

Rolfsen M. and C. Langeland (2012). "Successful maintenance practice through team autonomy", Employee Relations, 34 (3), 306-321.

Shah, Rachna and Peter T. Ward (2003). "Lean manufacturing: context, practice bundles, and performance", Journal of Operations Management, 21, 129-149.

Suh, N.P. (1990). "The principles of design". New York: Oxford Press.

Swanson, L. (1997). "An empirical study of the relationship between production technology and maintenance management", International Journal of Production Economics, 53 (2), 191-207.

Swanson, L. (2001). "Linking maintenance strategies to performance", International Journal of Production Economics 70 (3), 237-244.

Teeravaraprug Jiratat, Ketlada Kitiwanwong and Nuttapon Sae Tong (2011). "Relationship model and supporting activities of JIT, TQM and TMP", Songklanakaring Journal of Science and Technology, 33 (1), 101-106.

Theodorou, Petros and Giannoula Florou (2008). "Manufacturing strategies and financial performance-The effect of advanced information technology: CAD/CAM systems", International Journal of Management Science, 36, 107-121.

Thomas, Andrew and Gareth Lewis (2007). "Developing an SME-based integrated TPM-Six Sigma strategy", International Journal of Six Sigma and Competitive Advantage, 3 (3), 228-247.

Thomas F., Matthias Goetzfried and Prabir K. Basu (2010). "Analysis of the Implementation of Total Productive Maintenance, Total Quality Management, and Just-In-Time in Pharmaceutical Manufacturing", Journal of Pharmaceutical Innovation, 5,181-192.

Thun, J.H. (2008). "Supporting total productive maintenance by mobile devices", Production Planning \& Control, 19 (4), 430-434.

Van der Wal R.W.E. and D. Lynn (2002). "Total productive maintenance in a South African pulp and paper company: a case study", The TQM Magazine, 14 (6), 359-366.

Wang, F.K. and W. Lee (2001). "Learning curve analysis in total productive maintenance", Omega, 29, 491-499.

Yamashina, H. (1995). "Japanese manufacturing strategy and the role of total productive maintenance", Journal of Quality in Maintenance Engineering, 1 (1), 27-38. 